\title{
Severe Symmetric and Chronic Lower Eyelid Lymphedema in the Setting of Neck Surgery and Psoriasis
}

\author{
Michael E. Possin, Cat N. Burkat \\ Oculoplastics Service, Department of Ophthalmology and Visual Sciences, University of Wisconsin-Madison, Madison, USA. \\ Email: mepossin@gmail.com
}

Received September $7^{\text {th }}, 2012$; revised October $18^{\text {th }}, 2012$; accepted October $26^{\text {th }}, 2012$

\begin{abstract}
Purpose: To present a patient with bilateral severe and symmetric lower lid lymphedema in the setting of previous neck surgery and chronic psoriasis, and to review the potential relationships of neck surgery, irradiation, psoriasis, and rosacea to chronic lymphedema. Design: Single case report with literature review. Methods: A 60-year-old female with long-standing psoriasis presented with a 2-year history of severe, symmetric bilateral lower eyelid edema that developed after total laryngectomy and selective right neck dissection for recurrent Squamous Cell Carcinoma (SCC). 10 years prior she underwent radiation and radical left neck dissection for metastatic disease. Surgical management entailed transcutaneous debulking of the masses combined with ectropion repair and suture tarsorrhaphy. A comprehensive literature review was performed using Pubmed and Medline. Results: Surgical debulking of the soft tissue masses via a transcutaneous incision resulted in significant improvement in the patient's lymphedema without recurrence at 5 months follow-up. Histopathologic findings were consistent with chronic eyelid lymphedema. Conclusions: Isolated eyelid lymphedema is rare, with many etiologies, and poses a diagnostic challenge. While ophthalmologists are familiar with the ocular manifestations of rosacea such as conjunctivitis or blepharitis, it is important to consider rosacea as an etiology of eyelid lymphedema. Reviewing the history for previous surgery or radiation to the head and neck, or other dermatologic inflammatory disorders is also warranted. Rosaceous lymphedema is typically less severe than in post-surgical/radiation patients, and does not respond well to medical treatment; however, it often shows a favorable response to debulking blepharoplasty surgery, with or without skin grafting. This patient with a history of severe psoriasis and bilateral neck dissections with radiation for SCC also responded well to surgery without recurrence of lymphedema. Therefore, surgical debulking can be considered in these patients with severe eyelid lymphedema as an option to markedly improve visual function and overall cosmetic appearance.
\end{abstract}

Keywords: Lyphedema; Eyelid Edema; Psoriasis; Blepharoplasty; Rosacea; Eyelid Lymphatics; Facial Lymphatic System; Facial Edema; Squamous Cell Carcinoma

\section{Introduction}

Lymphedema occurs as a result of impairment of lymphatic drainage, leading to the accumulation of proteinrich lymphatic fluid within tissues. This protein-rich interstitial fluid leads to inflammation and an accumulation of fibroblasts, adipocytes and keratinocytes that transforms soft tissue into hard fibrotic tissue with stiff, thickened skin. The most common etiologies of head and neck lymphedema are associated with trauma, surgery, tumor growth, radiation and infection to the head and neck lymphatic system [1]. Isolated chronic eyelid lymphedema is rare, with only a few cases reported in the literature that are mainly associated with rosacea [1-16]. Lymphedema of the eyelids can be very debilitating to the patient, not only due to the physical appearance but also from visual field obstruction. This article presents a patient with severe lower eyelid lymphedema, reviews the lymphatic drainage considerations, and the potential association of certain dermatologic diseases with lymphedema.

\section{Materials and Methods}

This was a single case report. Informed consent was obtained from the patient, the guidelines of the Health Insurance Portability and Accountability Act were observed, and Institutional Review Board approval was obtained. Retrospective review of the medical record and a comprehensive review of the literature was performed using Pubmed and Medline, with key search words of: lymphedema, eyelid edema, rosacea, psoriasis, eyelid lymphatics, facial edema, facial lymphatic system, psoriatic arthritis. 


\section{Case Report}

A 60-year-old female presented with severe, painless, and symmetric bilateral eyelid edema of 2 years duration. 10 years earlier, she was diagnosed with metastatic squamous cell carcinoma of the left neck, for which she underwent combination chemotherapy and radiation treatment. She then underwent radical left neck dissection for residual disease that also involved the left jugular vein. She subsequently did well until recurrence 2 years prior to presentation, which required total laryngectomy, and selective right neck dissection. Postoperatively, she developed neck, facial, and lower eyelid lymphedema. The facial and neck edema resolved within 2 weeks, while her lower eyelid edema persisted.

On initial exam, the pupils, motility, and slit-lamp examinations were normal. The lower lids demonstrated large bilateral lower lid masses, measuring $5.5 \times 5 \mathrm{~cm}$ on the right and $6 \times 5 \mathrm{~cm}$ on the left, with anterior protrusion of $4.5 \mathrm{~cm}$. The masses were firm, with non-pitting edema and overlying brawny erythema and induration of the skin (Figure 1). Lower eyelid mechanical ectropion was also present due to the large masses. Blood work, including complete blood count, chemistries, creatinine, and thyroid panel, to evaluate for other systemic causes of eyelid edema was unremarkable. CT scan confirmed bilateral non-encapsulated lower lid soft tissue masses without extension into the orbit. Past medical history was also significant for severe psoriasis, which had led to multiple hospitalizations.

Surgical management involved bilateral sequential transcutaneous mass debulking with concurrent ectropion repair. Intraoperatively, there were no clear planes for dissection as the mass diffusely involved the skin, subcutaneous tissue, and orbicularis muscle layers (Figure 2(A)). Dissection was performed in the subcutaneous tissue plane to best delineate the anterior aspect of the mass. Posteriorly, dissection was taken to just anterior to the orbital septum. The soft tissue mass was placed in formalin for histopathologic evaluation. After horizontal tightening by a lateral tarsal strip procedure, the lower eyelid skin was draped superolaterally. The skin was markedly thickened and abnormal in texture and color (Figure 2(B)). The redundant skin removed measured over $5 \mathrm{~cm}$ vertically, and was submitted for pathology.
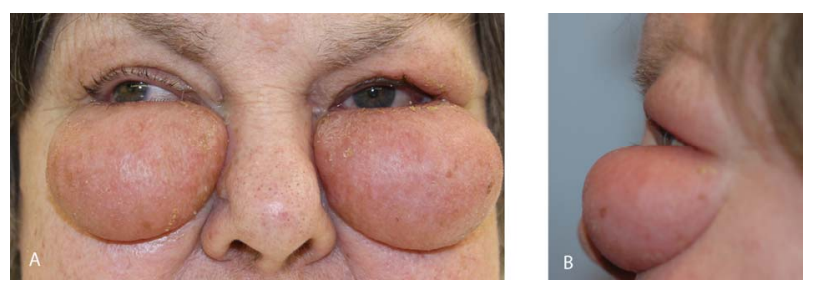

Figure 1. External photographs demonstrating severe bilateral lower eyelid masses: anterior view (A), and left lateral view (B).
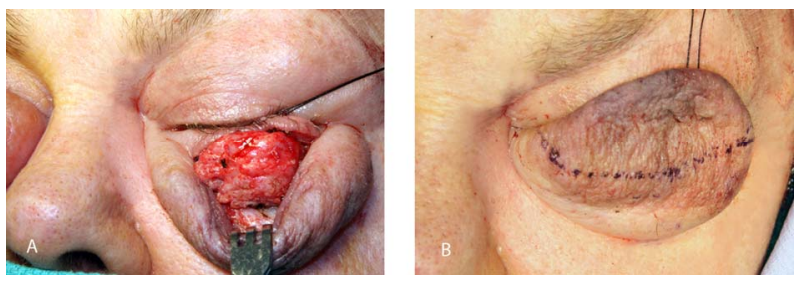

Figure 2. Intraoperative photographs of left lower eyelid surgery. (A) Soft tissue mass involving the skin, subcutaneous tissue, and orbicularis muscle layers dissected to the orbital septum, with overlying skin retracted inferiorly, (B) Redundant skin draped superiorly and marked for excision.

The skin was closed with 6-0 fast-absorbing plain gut suture, with no vertical lid tension. A frost suture tarsorrhaphy was placed for 2 weeks (Figure 3). At 5 days follow-up; there was a reduction in the eyelid lymphedema, with moderate postoperative swelling (Figure 4). Eyelid skin remained brawny and thickened in texture. Surgery was then performed on the contralateral side in the same fashion. At 5 months postoperatively, the thickened skin texture remained, but there was no recurrence of lymphedema, and she reported a significant improvement in vision and quality of life.

Histopathology of the excised skin revealed thickened eyelid skin demonstrating significant capillary proliferation, dermal fibrosis, reactive fibroblasts, and scattered areas of collagen breakdown. Multiple foci of chronic perivascular inflammation resembled follicular formation. A few non-caseating granulomas with mild inflammation were also seen. The deeper soft tissue mass demonstrated connective tissue with unremarkable skeletal muscle and adipose tissue. Similar to the skin, there was capillary proliferation, dermal fibrosis, collagen breakdown, and foci of chronic perivascular inflammation (Figure 5). Accessory lacrimal glands were also seen. The findings were consistent with chronic eyelid lymphedema.

\section{Discussion}

The differential diagnosis of chronic facial edema is expansive. The possible etiologies are inflammatory (acne vulgaris, systemic lupus erythematosus, sarcoidosis, allergic dermatitis, angioedema, dermatomyositis), infectious (erysipelas, mononucleosis, herpes zoster), congenital (facial hemiatrophy, Apert's syndrome, McCuneAlbright syndrome), malignant (angiosarcoma, lymphoma, mycosis fungoides, lymphosarcoma, myeloma, Kaposi's sarcoma), trauma, trichinosis, hypothyroidism and nephrotic syndrome [2]. Comprehensive discussion of these diseases and their systemic workup is beyond the scope of this article. Facial edema typically results as a complication of the overall disease, rather than presenting as isolated edema. The less common localized type of lymphedema usually results from previous infection, 


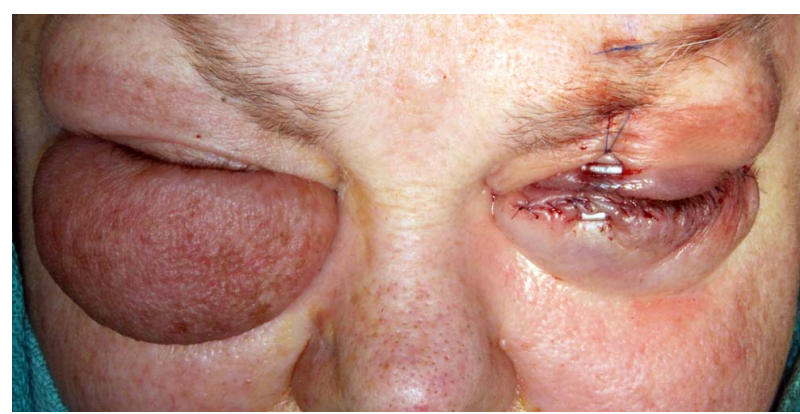

Figure 3. Immediate postoperative photograph with tarsorrhaphy in place, demonstrating significant debulking of the left lower eyelid mass through a subciliary incision.

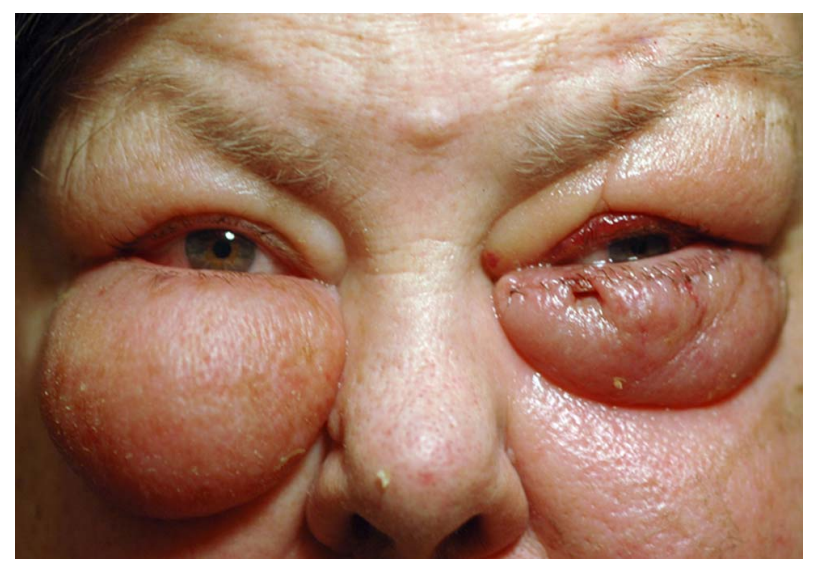

Figure 4. Postoperative photograph at 5 days follow up demonstrating reduction in left lower eyelid lymphedema, with moderate postoperative edema. Eyelid skin remained brawny and thickened in texture.

tumor growth, radiation, surgery, trauma, infection, or rosacea [1].

The severe bilateral eyelid edema seen in this patient developed postoperatively after total laryngectomy and selective right neck dissection two years prior to presentation. Before that, there was a remote history of left radical neck dissection with radiotherapy. It would first be important to consider whether the eyelid edema was secondary to a secondary or metastatic tumor. Although the CT images at the time of recurrence were not available from the outside facility, the report noted recurrence of disease in the right vocal cord. The left neck demonstrated normal postsurgical changes and no other abnormalities. She also underwent a PET scan prior to presentation that did not show any metastatic disease other than the hyperbolic activity in the right vocal cord. On presentation to our service regarding her eyelid issues, an additional CT of the head, maxiface, and orbits was performed which also confirmed no facial or orbital masses. The eyelid masses were localized in the preseptal eyelid tissues without orbital extension.

The histopathologic findings of the excised tissue were
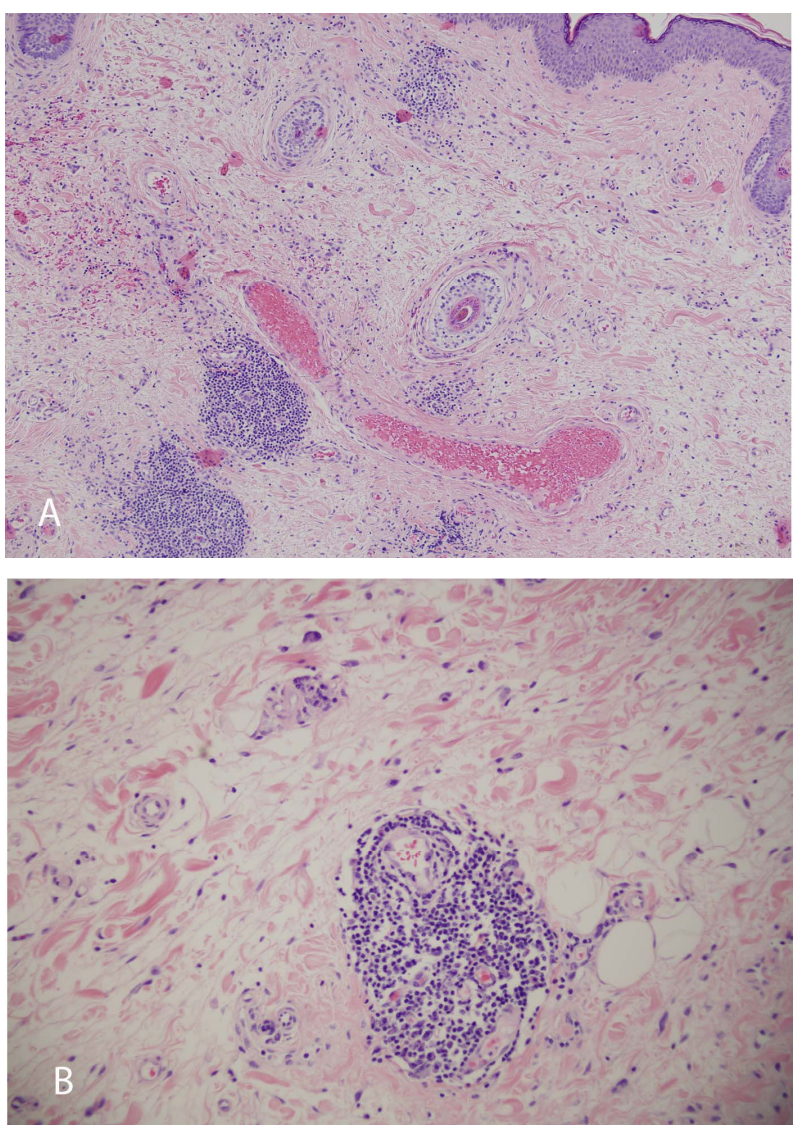

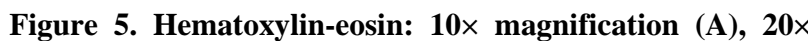
magnification (B). Histopathologic examination of the softtissue specimen demonstrates significant capillary proliferation, dermal fibrosis, reactive fibroblasts, collagen breakdown, and multiple foci of chronic perivascular inflammation resembling follicular formation. These changes are consistent with chronic lymphedema.

consistent with chronic lymphedema, which may have been secondary to disruption of the lymphatic system from tumor invasion, neck surgery, and/or radiation. However, it was interesting that the chronic lymphedema localized to the eyelids only, rather than throughout the face as well.

Although the eyelid lymphatic system is still not clearly understood, it is often believed to consist of two separate drainage systems, in which the submandibular lymph nodes drain the medial eyelids, central lower eyelid and cheek, while the parotid lymph nodes drain the upper eyelid and lateral aspects of both eyelids [17]. A recent study using lymphatic contrast and lymphangiograms in cadaver specimens demonstrated additional anatomic variation, with the lateral eyelid draining into either the parotid or submandibular nodes, and the medial eyelid draining into the submandibular, parotid or buccinator lymph nodes [18]. Two distinct levels of lymphatic vessels that are not interconnected have also been described in the lower lid. The superficial level is located 
between the skin and orbicularis muscle, while the deep system lies between the orbicularis and tarsal plate [19].

The parotid nodes also drain the frontal, and part of the parietal, regions of the head. The nasal, oral and mental regions of the scalp drain to the submandibular nodes and the oral and nasal branches drain to the buccinator lymph nodes. Given that the nodes that drain the eyelids also drain other regions of the head, it is curious that the lymphedema was solely isolated to the eyelids. In a study of 81 patients that received head and neck cancer treatment, only three patients developed edema in the face, neck and eyes, although details of tumor location, treatment, and severity of the eyelid edema were not given for comparison. No patients had isolated eyelid edema. Edema of the neck only or of the face and neck were most common and often improved, even with bilateral lymphatic disruption [20]. Other articles also report that secondary lymphedema after head and neck surgery is common, however, there are no reports of isolated eyelid edema $[21,22]$. Schiefkereported $17 \%-36 \%$ of patients had external lymphedema after selective neck dissection, but did not specify the location [23].

Two other cases of chronic bilateral eyelid lymphedema developing after surgery or radiation to the neck have been reported in the literature. Bernardini et al. presented a patient with a 9-month history of progressive lymphedema of the left upper and bilateral lower eyelids following right radical neck dissection with radiation for metastatic squamous cell carcinoma 5 years prior [2]. Clinically, the lymphedema appears similar to our patient; however, rosacea was reported as the cause of her eyelid edema rather than the neck surgery. In a series of 15 patients with chronic eyelid lymphedema, Chalasani et al. briefly reported a patient with 84 months of eyelid lymphedema after radiotherapy, although no further details were provided [3].

Two cases of unilateral eyelid lymphedema following surgery were also found in the literature. Jordandescribed a patient who developed right eyelid swelling following removal of a right parotid tumor, radical right neck dissection, and neck radiation [4]. Three months after neck irradiation, right upper and lower lid edema developed that improved with excision of the preseptal and pretarsal components of the orbicularis muscle. They concluded the lymphedema was secondary to damage to the parotid and submaxillary lymph nodes. Silverman et al. reported a case of severe unilateral, cheek and lower eyelid lymphedema after resection of an oropharyngeal tumor with radiation [5]. He was treated with surgical excision and split-thickness skin grafting.

Venous disruption may also be a contributing factor to the lymphedema in the patient presented here, as both internal jugular veins had been invaded with tumor. However, Ensari et al. reports that in patients who had staged bilateral neck dissections, with loss of the internal, external, posterior external and anterior jugular vein, postoperative digital subtraction angiography showed that venous drainage of the head was diverted from the jugular veins to the vertebral venous plexus [24]. Therefore, persistence of symmetric eyelid lymphedema would be unusual.

Certainly it is difficult to determine with any certainty if the lymphedema seen in our patient was more a result of the tumor removal, reconstruction surgery and radiation affecting lymphatic drainage, or if there was similarity to the cases presented in the literature with rosacea. It was initially assumed that neck surgery and irradiation were the causes; however, upon review of the literature as well as following consultation with head and neck surgeons, it appeared that although neck, laryngeal, or facial edema may be common, this is often transient due to robust collateral drainage. Isolated eyelid edema as seen in our patient is unusual and rare, even after significant bilateral neck surgery.

As mentioned previously, one of the two patients presented by Bernardini et al. had a prior history of neck surgery and radiation, but rosacea was considered to be the cause of lymphedema in both patients [2]. Rosacea is a cause of facial and eyelid edema, although is not often described in the ophthalmic literature. Ocular involvement in acne rosacea affects $3 \%-58 \%$ of patients, with $20 \%$ experiencing ocular manifestations as the initial presentation of rosacea [25]. The initial and most common stage of rosacea that is most familiar to ophthalmologists consists of erythema, teleangectasia, blepharitis, and conjunctivitis. The second stage includes papules and pustules, while the third stage includes inflammatory nodules, tissue hyperplasia, or rhinophyma. Lymphedema of the face or eyelids can present during any stage and is most often reported in the dermatologic literature [6-12] and only rarely in the ophthalmology literature $[2,3,13]$. Rosaceous edema is typically a non-pitting, bilateral, solid edema of the mid-third of the face, mainly affecting the periorbital tissues, but can also affect the forehead, glabella, nose or cheeks [6]. Unilateral eyelid lymphedema in association with rosacea has also been reported $[1,2,7,13]$. Facial erythema and telangiectasias on the nose and cheeks are often seen, in addition to the non-pitting eyelid edema [8].

Chalasani et al. reported fifteen patients with chronic eyelid lymphedema caused by: acne rosacea ( 9 patients), past radiotherapy (1 patient), trauma (1 patient), postvitrectomy silicone oil leak (1 patient), and unknown (4 patients) [3]. There were no measurements of the edema, but appeared less severe in degree. The patients were either observed, or treated with doxycycline or surgical debulking eyelid blepharoplasty. Both patients described by Bernardini et al. underwent debulking of the lower 
eyelid mass with horizontal eyelid tightening, with good functional improvement at three week follow up [2]. Maisels [14] and Silverman [5] reported successful results with excision of the lower eyelid masses and splitthickness skin grafting.

In contrast, Jamesreported a patient with rosacea and bilateral upper and lower eyelid edema that failed to respond to systemic steroids and tetracycline [15]. Excision and skin grafting of the affected lids was performed, with recurrence of lymphedema one year later. Marzanoalso described recurrence within a few months after upper eyelid blepharoplasty [6]. Kabir et al. reported a case of sudden onset, severe bilateral periorbital lymphedema of unknown etiology [16]. Two episodes of acute edema occurred after staged debulkings, and they concluded that using a skin graft offered a better cosmetic outcome.

Uhara et al. described a 53-year old man with symmetrical non-pitting edema, conjunctivitis, and acneiform eruptions on the face with biopsies consistent with rosacea [9]. He was treated with fleroxacin $(100 \mathrm{mg} /$ day $)$ for two weeks with a marked reduction of both facial and periorbital edema. In contrast, most cases of chronic eyelid lymphedema in patients with acne rosacea in the dermatology literature did not improve with medical treatment [10-12].

The pathogenesis of rosaceous lymphedema is unclear, but chronic inflammation is believed to play a role. Lymphostasis promotes an influx of inflammatory cells and release of mediators and cytokines by activated endothelial cells, lymphocytes and macrophages [26]. Due to the lymphatic dysfunction, these cytokines and chemokines, which normally would be cleared from the interstitium, remain in the tissue, recruiting more inflammatory cells. This chronic inflammatory state activates fibroblasts, which destroy elastin that surrounds blood vessels, leading to transudation of fluids. Also, the replacement of elastin with collagen causes fibrosis, which may obstruct the lymphatic vessels of the deep dermis, leading to congestion, fluid accumulation and lymphedema [27]. This is supported by the histologic findings in rosaceous lymphedema, which include dilated blood vessels, perifollicular fibrosis, perivascular lymphocytes, histiocytes, neutrophils, perilymphatic granulomas and stromal edema $[2,7,27]$. Although clinically the presentation of our patient looked most similar to the two patients with rosacea presented by Bernardini [2], our patient did not have any signs or symptoms of rosacea. However, her history of psoriasis led us to question whether the inflammatory component and histopathologic changes found in rosacea that lead to this lymphedematous manifestation could also be seen in other dermatologic disorders such as psoriasis. Histopathologic examination of psoriatic skin lesions has demonstrated abnormalities of the lymphatics such as dilatation and lack of fenestration
[28]. To our knowledge there are no reports in the literature of psoriasis causing facial or eyelid lymphedema, but reports of lymphedema in the extremities, predominantly involving the upper limbs, are common in association with psoriatric arthritis [29-32]. 11\% of people with psoriasis may eventually develop psoriatic arthritis, and our patient had a known history of severe psoriasis, as well as arthritis of the hands, wrists and neck [33].

The exact pathogenesis of psoriatric arthritis lymphedema is unknown, but similar to rosacea, inflammation plays a role. Yamamoto et al. suggests that the inflammatory products from the synovium are deposited in the adjacent lymphatics, leading to the lymphangitis and lymphatic obstruction [29]. Quantitative lymphoscintigraphy disclosed abnormal lymphatic drainage of the affected edematous limb [30], whereas another study found no significant change in lymphatic flow in patients with rheumatoid or psoriatic arthritis [31].

In conclusion, isolated eyelid lymphedema is rare, with many etiologies, and poses a diagnostic challenge to the physician. While ophthalmologists are familiar with the ocular manifestations of rosacea such as conjunctivitis or blepharitis, it is important to consider rosacea as a possible etiology of significant eyelid lymphedema. A complete review of history for previous neck surgery or radiation to the head and neck regions, or other dermatologic inflammatory disorders may also be warranted. To our knowledge, there is no previous report of eyelid lymphedema associated with psoriasis. In general, rosaceous lymphedema is typically less severe than in postsurgical/radiation patients, and does not respond well to medical treatment; however, it often shows a favorable response to debulking blepharoplasty surgery, with or without skin grafting. This patient with a history of both severe psoriasis and bilateral neck surgery and radiation for malignancy of the neck also responded well to surgery without recurrence of lymphedema. Therefore, surgical debulking can be considered in these patients with severe eyelid lymphedema as an option to markedly improve vital visual function as well as overall cosmetic appearance.

\section{Acknowledgements}

Supported in part by an unrestricted grant to the University of Wisconsin Department of Ophthalmology and Visual Sciences from the Research to Prevent Blindness, Inc., New York, NY.

\section{REFERENCES}

[1] S. Lu, T. Tran, D. Jones, et al., "Localized Lymphedema (Elephantiasis): A Case Series and Review of the Literature," Journal of Cutaneous Pathology, Vol. 36, No. 1, 2008, pp. 1-20. doi:10.1111/j.1600-0560.2008.00990.x 
[2] F. P. Bernardini, R. C. Kersten, L. M. Khouri, et al., "Chronic Eyelid Lymphoedema and Acne Rosacea. Report of Two Cases," Ophthalmology, Vol. 107, No. 12, 2000, pp. 2220-2223. doi:10.1016/S0161-6420(00)00429-2

[3] R. Chalasani and A. McNab, "Chronic Lymphedema of the Eyelid: Case Series," Orbit, Vol. 29, No. 4, 2010, pp. 222-226. doi:10.3109/01676831003695735

[4] D. R. Jordan, "Eyelid Lymphedema," Arch Opthalmology, Vol. 109, No. 2, 1991, pp. 178-179. doi:10.1001/archopht.1991.01080020024015

[5] A. T. Silverman, R. Hoffman, M. Cohen and R. Garza, "Severe Cheek and Lower Eyelid Lymphedema after Resection of Oropharyngeal Tumor and Radiation," Journal of Craniofacial Surgery, Vol. 21 No. 2, 2010, pp. 598601. doi:10.1097/SCS.0b013e3181d08c90

[6] A. V. Marzano, P. Vezzoli and E. Alessi, "Elephantoid Oedema of the Eyelids," Journal of the European Academy of Dermatology and Venereology, Vol. 18, No. 4, 2004, pp. 459-462. doi:10.1111/j.1468-3083.2004.00941.x

[7] D. M. Chen and D. L. Crosby, "Periorbital Edema as an Initial Presentation of Rosacea," Journal of the American Academy of Dermatology, Vol. 37, No. 2, 1997, pp. 346348. doi:10.1016/S0190-9622(97)80389-1

[8] A. Morales-Burgos, G. Alvarez Del Manzano, J. L. Sánchez and C. L. Cruz, "Persistent Eyelid Swelling in a Patient with Rosacea," Puerto Rico Health Sciences Journal, Vol. 28, No. 1, 2009, pp. 80-82.

[9] H. Uhara, S. Kawachi and T. Saida, "Solid Facial Oedema in a Patient with Rosacea," The Journal of Dermatology, Vol. 27, 2000, pp. 214-216.

[10] B. F. O'Donnell, "Visual Impairment Secondary to Rosacea," British Journal of Dermatology, Vol. 127, No. 3, 1992, pp. 300-301. doi:10.1111/j.1365-2133.1992.tb00136.x

[11] T. Jansen and G. Plewig, "The Treatment of Rosaceous Lymphoedema," Clinical and Experimental Dermatology, Vol. 22, 1997, pp. 54-64.

[12] D. Harvey, N. A. Fenske and L. F. Glass, "Rosaceous Lymphedema: A Rare Variant of a Common Disorder," $\mathrm{Cu}$ tis, Vol. 61, 1998, pp. 321-324.

[13] T. F. Lai, I. Leibovitch, C. James, et al., "Rosacea Lymphoedema of the Eyelid," Acta Ophthalmologica Scandinavica, Vol. 82, No. 6, 2004, pp. 765-767. doi:10.1111/j.1600-0420.2004.00335.x

[14] D. O. Maisels and A. O. A. Korachi, "Lymphoedema of the Eyelids in the Yellow Nail Syndrome," British Journal of Plastic Surgery, Vol. 38, No. 1, 1985, pp. 93-96. doi:10.1016/0007-1226(85)90093-1

[15] J. H. James, "Lymphedema of the Eyelids," Plastic and Reconstructive Surgery, Vol. 61, No. 2, 1978, pp. 703706. doi:10.1097/00006534-197805000-00007

[16] S. M. Kabir, A. Raurell and V. Ramakrishnan, "Lymphoedema of the Eyelids," British Journal of Plastic Surgery, Vol. 55, No. 2, 2002, pp. 153-154. doi:10.1054/bjps.2001.3763

[17] K. W. Dewey, "A Contribution to the Study of the Lymphatic System of the Eye," The Anatomical Record, Vol. 19,

\section{No. 2, 1920, pp. 125-140. doi:10.1002/ar.1090190205}

[18] W. R. Pan, C. M. Le Roux and C. A. Briggs, "Variations in the Lymphatic Drainage Pattern of the Head and Neck: Further Anatomic Studies and Clinical Implications," Plastic and Reconstructive Surgery, Vol. 127, No. 2, 2011, pp. 611-620. doi:10.1097/PRS.0b013e3181fed511

[19] B. E. Cook, M. J. Lucarelli, B. N. Lemke, et al., "Eyelid Lymphatics. I. Histochemical Comparisons between the Monkey and Human," Ophthalmic Plastic \& Reconstructive Surgery, Vol. 18, No. 1, 2002, pp. 18-23. doi:10.1097/00002341-200201000-00004

[20] J. Deng, S. H. Ridner, M. S. Dietrich, et al., "Prevalence of Secondary Lymphedema in Patients with Head and Neck Cancer," Journal of Pain and Symptom Management, in press.

[21] J. Buntzel, M. Glatzel, R. Mucke, et al., "Influence of Amifostine on Late Radiation-Toxicity in Head and Neck Cancer-A Follow-Up Study," Anticancer Research, Vol. 27, No. 4A, 2007, pp. 953-956.

[22] H. A. Wolff, T. Overbeck, R. M. Roedel, et al., "Toxicity of Daily Low Dose Cisplatin in Radiochemotherapy for locally Advanced Head and Neck Cancer," Journal of Cancer Research and Clinical Oncology, Vol. 135, 2009, pp. 961-967. doi:10.1007/s00432-008-0532-x

[23] F. Schiefke, M. Akdemir, A. Weber, et al., "Function, Postoperative Morbidity, and Quality of Life after Cervical Sentinel Node Biopsy and after Selective Neck Dissection," Head and Neck, Vol. 31, No. 4, 2009, pp. 503512. doi:10.1002/hed.21001

[24] S. Ensari, E. Kaptanoğlu, K. Tun, et al., "Venous Outflow of the Brain after Bilateral Complete Jugular Ligation," Turkish Neurosurgery, Vol. 18, No. 1, 2008, pp. 56-60.

[25] D. J. Browning and A. D. Proia, “Ocular Rosacea," Survey of Ophthalmology, Vol. 31, 1986, pp. 145-158. doi:10.1016/0039-6257(86)90034-2

[26] J. Daroczy, "Pathology of lymphedema," Clinics in Dermatology, Vol. 13, 1995, pp. 433-444. doi:10.1016/0738-081X(95)00086-U

[27] J. K. Wilkin, "Rosacea. Pathophysiology and Treatment," Archives of Dermatology, Vol. 130, No. 3, 1994, pp. 359362. doi:10.1001/archderm.1994.01690030091015

[28] I. M. Braverman and A. Yen, "Microcirculation in Psoriatic Skin," Journal of Investigative Dermatology, Vol. 62, 1974, pp. 493-502. doi:10.1111/1523-1747.ep12681007

[29] T. Yamamoto and K. Nishioka, "Psoriasis Arthropathy and Lymphedema," The Journal of Dermatology, Vol. 29, 2002, pp. 812-814.

[30] M. Bohm, B. Riemann, T. A. Luger and G. Bosmann, "Bilateral Upper Limb Lymphoedema Associated with Psoriatic Arthritis: A Case Report and Review of the Literature," British Journal of Dermatology, Vol. 143, 2000, pp. 1297-1301. doi:10.1046/j.1365-2133.2000.03905.x

[31] P. D. W. Kiely, A. E. A. Joseph, P. S. Mortimer and B. E. Bourke, "Upper Limb Lymphedema Associated with Polyarthritis of Rheumatoid Type," The Journal of Rheumatology, Vol. 21, 1994, pp. 1043-1045. 
[32] L. Quarta, A. Corrado, F. d'Onofrio, et al., "Two Cases of Distalextremityswelling with Pittingoedema in Psoriatic Arthritis: The Different Pathological Mechanisms," Rheumatology International, Vol. 30, No. 10, 2010, pp. 1367-1370. doi:10.1007/s00296-009-1060-y

[33] J. M. Gelfand, D. D. Gladman, P. J. Mease, et al., "Epi- demiology of Psoriatic Arthritis in the Population of the United States," Journal of the American Academy of Dermatology, Vol. 53, No. 4, 2005, p. 573. doi:10.1016/i.jaad.2005.03.046 